\title{
ESTUDIO COMPARATIVO DE LA AUTONOMIA FUNCIONAL DE ADULTOS MAYORES: ATLETAS Y SEDENTARIOS, EN ALTITUD MODERADA
}

\section{COMPARATIVE STUDY OF FUNCTIONAL AUTONOMY IN ATHLETE AND SEDENTARY OLD MEN, IN MODERATE ALTITUDE}

\author{
Cesar Giovanni Quintero-Burgos ${ }^{l}$ \\ Victor Manuel Melgarejo-Pinto ${ }^{2}$ \\ Juan Manuel Ospina-Díaz \\ Universidad Pedagógica y Tecnológica de Colombia. Tunja, \\ Colombia \\ vic.melgarejo@live.com
}

RESUMEN

La práctica sistemática de actividad física contribuye al mejoramiento de las condiciones de vida y salud y retarda el proceso de envejecimiento del adulto mayor. Objetivo. Identificar diferencias en la autonomía funcional en hombres de 60 a 70 años, practicantes de ciclismo y sedentarios, residentes en Tunja, a altitud moderada. Materiales y Métodos. Estudio comparativo de corte transversal analítico. Participaron 22 adultos mayores, de sexo masculino; 11 sedentarios y 11 practicantes de ciclo montañismo, con edades entre 60 y 70 años (media $63.03 \pm 4.32$ años) previo consentimiento informado se sometieron a evaluación cineantropométrica por bioimpedancia, las pruebas de la batería Senior Fitness Test (SFT) de Rikli y Jones (1999a) el Test de Ästrand submaximal y dinamometría manual derecha e izquierda. Resultados: Los grupos se encontraron comparables en edad, peso, talla e índice de masa corporal ( $p>0,05)$. Se registraron diferencias estadísticamente significativas a favor del grupo de ciclo montañistas, en la media del porcentaje de grasa corporal, densidad ósea, masa muscular, mientras que ni el índice de masa corporal $(25,6 \pm 3,3$ y $25,2 \pm 2,5)$ registraron diferencias significativas. En la prueba SFT se encontraron diferencias estadísticamente significativas en la flexión de cadera $(p<0,001)$. En la prueba submaximal se encontraron diferencias estadísticamente significativas en la prueba de esfuerzo en Watts $(\mathrm{p}=0,021)$. En la dinamometría de la mano izquierda también se encontraron diferencias estadísticamente significativas $(\mathrm{p}=0,0007)$. Conclusión. Los adultos mayores que adelantan rutinariamente prácticas de ejercicio físico como el ciclo montañismo presentan mejores características de composición corporal, flexibilidad de la cintura, mejor capacidad aeróbica y motricidad.

Palabras claves: actividades físicas, adulto mayor, autonomía personal

\section{SUMMARY}

The systematic practice of physical activity contributes to improve living and health conditions and slows dawn the aging process in seniors. Objective. Identify differences in functional autonomy in 60-70 year old mountain cyclists and sedentary males residing in Tunja, at moderate height. Materials and Methods. Comparative analytical cross-sectional study. A total of 22 elderly males participated; 11 sedentary seniors and 11 mountain cyclists, ages between 60 and 70 (mean $63.03 \pm 4.32$ years). Subjects agreed to take the Kinanthropometric assessment using a bioimpedance analysis, the Senior Fitness Test (SFT) battery developed by Rikli and Jones (1999a), the submaximal Ästrand Test and the Right and Left Handgrip 
Dynamometer Test. Results: Groups were comparable in age, weight, height and body mass index ( $\mathrm{p}>$ 0.05). Statistically significant differences were recorded in favor of the mountain cyclist group, in the mean percentage of body fat, bone density, and muscle mass; Statistically significant differences were found in the SFT test, specifically in hip flexion $(\mathrm{p}<0.001)$ and in the submaximal test in the stress test in Watts $(\mathrm{p}=0.021)$. Statistically significant differences were also found in the left hand during the dynamometry test. $(\mathrm{p}=0.0007)$. Conclusion. Seniors who routinely practice physical activities, such as mountain biking, show better indicators of health in body composition, waist flexibility, aerobic capacity and motor skills.

Keywords: Physical Activities, Elder people, Personal autonomy (DeCS)

\section{Introducción}

El envejecimiento humano es un fenómeno universal e inevitable, la esperanza media de vida ha mejorado en el último siglo según la ONU, el número de personas mayores de 60 años aumentó del 8 al 10\% (un 2\%) entre 1950 y 2000, del de la población total, también se prevé que el número de personas de edad avanzada aumentará hasta el $22 \%$ de la población total en el 2050 (ONU, 2005); así como la Revolución Industrial surgida en el siglo XVIII, que propició mejores condiciones de trabajo, salarios suficientes y nuevas políticas en materia de salud, lo logrado hasta el momento en este aspecto, ha estimulado incluso en los países en vías de desarrollo, un incremento en la esperanza de vida al nacer, que en la actualidad puede estar por encima de los 75 años en promedio para América Latina (Chávez, et al, 2004). Para De Rosnay (1988) el envejecimiento es un proceso natural del organismo del ser humano influenciado por factores que ocurren con el paso del tiempo y en el que se hacen evidentes cambios funcionales, fisiológicos, biológicos, sociales y mentales. En esta etapa de la vida ocurre un decremento de la actividad física a la vez que también disminuye la intensidad y la frecuencia de realización de las tareas rutinarias ligadas al trabajo (Moreno 2005). La práctica rutinaria de algún tipo de actividad física y del ejercicio físico son un mecanismo eficaz y apropiado para mantener buenos niveles de capacidad motriz, que podría impactar sobre la disminución de las capacidades físicas y de las actividades de la vida diaria, por consiguiente, un nivel adecuado de actividad física debe retrasar la pérdida de habilidades funcionales y físicas y ayudar a mantener un estilo de vida saludable para las personas mayores. (Del Pozo, 2013; Milanović, 2013; Martín, et al, 2002) al evaluar la composición corporal en adultos mayores, demuestran la alta prevalencia de afectaciones en el ambiente bioquímico, los porcentajes de grasa y la densidad ósea y disminución de la masa muscular en esta población. Por esta razón, evaluar la condición física del adulto mayor es indispensable, al constituirse como un indicador confiable del estado de salud general. Al respecto, la Organización Mundial de la Salud (OMS, 2010) dentro de las recomendaciones mundiales sobre actividad física para la salud, indica que una de las mejores formas de medir la salud de los adultos mayores es mediante las valoraciones de salud cardiorrespiratoria, salud metabólica y salud del aparato locomotor, entre otras. Se encuentra un creciente interés por desarrollar rutinariamente pruebas de evaluación de la capacidad física, y la integridad neuromuscular, con el propósito de sopesar su empleo potencial 
como herramienta sencilla en la detección de alteraciones y predicción de riesgo en población Adulta mayor e individualizar en grupos de pacientes, para evaluar respuestas a tratamientos. De hecho, las mediciones de la fuerza muscular y la capacidad funcional locomotora se han correlacionado con la morbilidad, la independencia funcional y la mortalidad en el adulto mayor (Cooper 2010); Porter (2006) y Salazar (2013) concuerdan al señalar que aunque la fuerza es importante, parece ser que la potencia tiene una mayor relevancia en la mejora de las tareas cotidianas, especialmente la acción de caminar, siendo esta la razón principal para incluir el entrenamiento de la potencia como parte de un programa en el adulto mayor, ya que en la actividad cotidiana se requiere de movimientos fuertes y rápidos. Gomes de Souza, et al, (2002) demostraron que el entrenamiento de fuerza realizado de 2 a 3 series de 8 a 10 repeticiones, con 70 a $80 \%$ de $1 \mathrm{RM}$, por 8 semanas, frecuencia semanal de 3 sesiones, proporciona cambios positivos $(\mathrm{p}<0,05)$ en el aspecto físico/ funcional y psicológico/autoestima en adultos mayores (X 66,3 $\pm 7,84$ de edad). el programa correspondió 2 a 3 series de 8 a 10 repeticiones, con 70 a 80\% de 1RM, por ocho semanas.

Por otro lado, la habituación al desarrollo de actividades sedentarias prolongadas, especialmente ver televisión, se han encontrado asociada con resultados adversos para la salud, independientemente de los niveles de actividad física, con Riesgo Relativo $\mathrm{RR}=1,13\left(\mathrm{IC}_{95 \%}: 1,07\right.$ 118; $\mathrm{p}<0,01)$, (Grøntved \& Hu 2011); en consecuencia, el comportamiento sedentario se considera ahora como una categoría o factor, que puede suponer un riesgo para el ejercicio del derecho a la salud. Sin embargo, la mayoría de investigaciones adelantadas hasta la fecha se han centrado en los resultados de afectación cardiaca y metabólica, además de los resultados de mortalidad, de manera que el papel independiente de la conducta sedentaria para explicar la disminución de la fuerza muscular en relación con el envejecimiento permanece poco analizado (Hamer \& Stamatakis 2013). Se ha demostrado que para los adultos mayores, la realización constante de actividad física es beneficiosa para mantener y mejorar el estado general de salud, siendo recomendable que los adultos practiquen durante casi todos los días un promedio de treinta minutos de actividad física moderada; para los adultos mayores esto es equivalente a caminar a paso ligero a una velocidad entre 3-6 millas por hora. A pesar de estas recomendaciones, muchos adultos, especialmente de mediana edad y mayores, prefieren mantener un estilo de vida sedentario; investigaciones adelantadas por el Center for Disease Control and Prevention (CDC) de Atlanta en 2008 revelaron que en los Estados Unidos de América (EE.UU) aproximadamente 1 de cada 5 (21\%) adultos cumple con las Guías de Actividad Física, lo que equivale a decir que más del 60\% de los adultos no participan en estos programas unos estatales y otros ofrecidos por la empresa privada u organismos de salud; y que aproximadamente el 25\% no están activos en absoluto, es decir la inactividad física es más común. Carlson et al. (2015) afirman que "los niveles de actividad física no son adecuados para las directrices actuales y están asociados con una carga financiera significativa para la salud de los EE.UU., encontrando que la actividad física inadecuada se asocia con un porcentaje significativo de los gastos de atención médica". (p 316)

Se han diseñado protocolos y baterías de test para evaluar la aptitud y condición física y autonomía funcional del adulto mayor con fines de asistencia; los más conocidos en el ambiente 
latinoamericano, entre otros, son: 1. Indice de Bartel que por cuestionario con valores asignados a cada ítem se basan en el tiempo y la cantidad de asistencia física real requerida si un paciente es o no de realizar la actividades de la vida diaria AVD (Mahoney \& Bartel 1965). 2. La batería Senior Fitness Test (SFT) diseñada por Rikli, \& Jones 1999a, con fines de evaluación de la aptitud física mediante seis pruebas de resistencia, fuerza y flexibilidad. y 3. El Protocolo GDLAM, diseñado por Martín Dantas constituido por - una prueba de 10m caminando (C10M), - levantarse de una posición sentada (LPS), - pasando de posición de decúbito prono (LPDV) y levantarse de la silla y moverse en la casa (LCLC) (Martin, et al. 2005).Por las consideraciones expuestas, en el presente estudio se propuso evaluar, a través de la determinación de diferencias y similitudes en cuanto a autonomía funcional y variables antropométricas, el impacto de la práctica de actividad física moderada a intensa, en un grupo de hombres de entre 60 y 70 años, adultos mayores practicantes de ciclo-montañismo, comparados con un grupo control de personas con bajo nivel de actividad física, residentes en la altitud moderada de la ciudad de Tunja, Boyacá (2750msn), según clasificación de altitudes de Bartsch \& Saltin (2008).

\section{Materiales y métodos}

Se diseñó un estudio observacional comparativo de corte transversal; se definió como población objetivo a dos grupos de adultos mayores de sexo masculino; el grupo sedentario conformado por 36 personas con edades entre los 60 y 70 años vinculados laboralmente a la Universidad Pedagógica y Tecnológica de Colombia, sede Tunja, como docentes, empleados o trabajadores, registrados en la oficina de Talento Humano de la UPTC de Tunja, en marzo de 2013 (UPTC 2013); - el grupo de practicantes de ciclo montañismo estaba integrado por 29 integrantes del Club de ciclo montañismo DONATO ILB con sede en la ciudad de Tunja, Boyacá; mediante muestreo secuencial aleatorio por conveniencia se reclutó para conformar cada grupo a once participantes, acorde con los criterios de inclusión consignados en el protocolo. Cada uno de los participantes fue informado ampliamente de los propósitos del estudio así como del tipo de pruebas a adelantar; si estaba de acuerdo con su participación se le pidió que signara un formato de consentimiento informado; el estudio en su totalidad fue evaluado y aprobado por un Comité de Ética en investigación institucional.

Los parámetros de peso y composición corporal fueron evaluados en una báscula Tanita Ironman Body Composition BC549 ${ }^{\mathrm{TM}}$, que permite hacer estimaciones sobre la composición corporal mediante análisis de Impedancia Bioeléctrica; para ello las almohadillas de la plataforma de análisis hacen pasar señales eléctricas de baja intensidad sobre el cuerpo, que atraviesan libremente la materia líquida de los músculos y otros tejidos, pero encuentran resistencia al atravesar el tejido graso, dada la escasez de fluido; esta resistencia es lo que se denomina impedancia. A partir del valor de impedancia registrado, un software especial estima el porcentaje de grasa y músculo corporal. Estos cálculos se han originado en estudios científicos que desarrollan ecuaciones validadas mediante métodos estadísticos (Martin, et al, 2001). 
A cada uno de los participantes se le practicó la batería Senior Fitness Test (SFT) diseñada por Rikli \& Jones (1999b), test que surgió de la necesidad de disponer de una herramienta que permitiese valorar de forma práctica y con seguridad la condición física funcional de los adultos mayores, definida como la capacidad física para desarrollar actividades normales de la vida diaria de forma segura, con independencia y sin una excesiva fatiga (Rikli, \& Jones 1999a). La batería de pruebas SFT es muy completa, los test que componen la batería recogen el mayor número de componentes del fitness asociados con la independencia funcional, puede realizarse en personas con diferentes edades en el rango entre 60 y 94 años de edad y con diversos niveles de capacidad física y funcional, ya que esta batería cubre un amplio rango de capacidad funcional, desde los más frágiles hasta los físicamente muy funcionales; es de fácil aplicación en cuanto al equipamiento y espacio necesarios, por lo que puede realizarse fuera del laboratorio y tiene valores de referencia expresados en percentiles para cada uno de los test, permitiendo comparar los resultados entre personas del mismo sexo y edad.

Adicionalmente, mediante el test de Ästrand, que permite estimar la capacidad aeróbica a partir de los valores de la tasa de pulso y el consumo de oxígeno durante un test de trabajo con esfuerzo submaximal con bicicleta, banda de marcha o conteo de pasos, se estimaron los correspondientes parámetros para los dos grupos (Ästrand, \& Rodahl, 1992; Hoehn, et al, 2015). Finalmente, se practicó una dinamometría en ambas manos a todos los participantes, prueba que tiene por objeto medir la potencia (fuerza estática) de los músculos flexores de la mano y el antebrazo, izquierdo y derecho; en los adultos mayores es muy útil probar la fuerza de prensión manual, para lo cual se emplea el dinamómetro. La dinamometría es una medida confiable, por lo que se ha estandarizado para uso clínico y en investigación. Se encontró una correlación de 0.68 entre el índice de Barthel y la fuerza prensil; es una valoración rápida y de bajo costo. El uso de la fuerza prensil como un modelo para describir en general los cambios en la fuerza se sustenta en su correlación significativa con otras medidas de fuerza (Campo 2006)

Con los datos obtenidos mediante las estimaciones se ensambló una base de datos, la cual posteriormente se analizó utilizando el paquete estadístico Epi-Info $7^{\circledR}$. Se adelantó inicialmente un análisis de normalidad con shapiro-wilks para establecer la comparabilidad de los grupos $1(\mathrm{p}=0,057)$ y $2(\mathrm{p}=0,263)$ indicando con $\mathrm{p} \geq 0,05$ que cumplen con distribución normal. posteriormente se evaluaron los resultados de los diferentes test aplicados a cada uno de los grupos. Las variables analizadas por separado se reportaron estableciendo las correspondientes medidas de tendencia central y dispersión para análisis de normalidad con $\mathrm{t}$ de student para muestras independientes con $\mathrm{n}<30$, datos continuos, además de los intervalos de confianza al 95\% para las variables discretas. Posteriormente se adelantaron comparaciones de los dos grupos, validando las correspondientes hipótesis mediante la prueba $t$ de student $n<30$ para muestras independientes en las pruebas: 1. sentarse y levantarse de una silla, 2. Levantarse, caminar y volver a sentarse, 3. Flexión de cadera, 4. Curl de brazos, y 5. Juntar las manos espalda. 6. Test de Ästrand, prueba de esfuerzo y 7. Dinamometría manual derecha e izquierda, en variables continuas, dado el tamaño de la muestra y el desconocimiento de la varianza poblacional; 


\section{Resultados}

El grupo estudiado estuvo conformado por 22 adultos mayores, varones, con edad promedio de 63,36 años ( $\mathrm{SD}=2,55$; rango 60 - 69 años; $\mathrm{p}=0,62)$, media de peso 69,97 $\mathrm{Kg}(\mathrm{SD}=8,89, \mathrm{p}=0,6)$; talla promedio 1,65 m. (SD 0,06, p=0,65); media de Índice de Masa Corporal (IMC) 25,4 (SD=2,9, $\mathrm{p}=0,76$ ); en la tabla 1 se presentan los datos antropométricos de las muestras: General, adulto mayor atleta y sedentario; se encontró que no hay diferencias estadísticamente significativas de las variables anteriores, por lo cual se considera que los dos grupos son comparables.

Tabla 1

Adultos mayores, Tunja, Colombia.

Datos antropométricos de ambos grupos en estudio 2013

\begin{tabular}{ccccc}
\hline Variable & $\begin{array}{c}\text { General } \\
(\mathrm{N}=22)\end{array}$ & $\begin{array}{c}\text { Adulto Mayor } \\
\text { Ciclista } \\
(\mathrm{N}=11)\end{array}$ & $\begin{array}{c}\text { Adulto Mayor } \\
\text { Sedentario } \\
(\mathrm{N}=11)\end{array}$ & $\begin{array}{c}\mathrm{p} \mathrm{tde} \\
\text { (student })\end{array}$ \\
\hline $\begin{array}{c}\text { Edad } \\
\text { (M y Ds) }\end{array}$ & $63,3 \pm 2,55$ & $63 \pm 1,9$ & $63,3 \pm 3,1$ & 0,62 \\
$\begin{array}{c}\text { Peso } \\
\text { (M y Ds) }\end{array}$ & $69,97 \pm 8,89$ & $70,9 \pm 9,4$ & $68,9 \pm 8,6$ & 0,6 \\
$\begin{array}{c}\text { Talla } \\
\text { (M y Ds) }\end{array}$ & $1,65 \pm 0,06$ & $1,66 \pm 0,07$ & $1,65 \pm 0,06$ & 0,65 \\
$\begin{array}{c}\text { IMC } \\
\text { (M y Ds })\end{array}$ & $25,4 \pm 2,9$ & $25,6 \pm 3,3$ & $25,2 \pm 2,5$ & 0,76 \\
\hline
\end{tabular}

Tabla 2

Adultos mayores, Tunja, Colombia.Valores promedio de la composición corporal

\begin{tabular}{cccc}
\hline Prueba & $\begin{array}{c}\text { Adulto mayor } \\
\text { ciclista }\end{array}$ & $\begin{array}{c}\text { Adulto mayor } \\
\text { sedentario }\end{array}$ & $\mathrm{p}$ (t de student) \\
\hline $\begin{array}{c}\text { \% Grasa } \\
\text { (M y Ds) }\end{array}$ & $20,1 \pm 3,74$ & $25,1 \pm 4,76$ & $0,013^{*}$ \\
$\begin{array}{c}\text { \% Oseo } \\
\text { (M yDs) }\end{array}$ & $28,36 \pm 2,58$ & $26,09 \pm 2,11$ & $0,035^{*}$ \\
\% Muscular & $53,67 \pm 5,48$ & $48,1 \pm 4,28$ & $0,018^{*}$ \\
$\begin{array}{c}\text { (M yDs) } \\
\text { \% Agua }\end{array}$ & $53,51 \pm 13,91$ & $53,87 \pm 3,48$ & 0,95 \\
$\begin{array}{c}\text { (M y Ds) } \\
\text { Indice Cintura- } \\
\text { Cadera }\end{array}$ & $0,973 \pm 0,047$ & $1,006 \pm 0,043$ & 0,091 \\
\hline
\end{tabular}

$* \mathrm{P}<0,05$ 
En la evaluación de la estructura corporal se encontró similar grado de composición en los porcentajes de agua corporal $(\mathrm{p}=0,95)$ y en el Índice Cintura-Cadera (ICC, $\mathrm{p}<0,09)$, mientras que se hallaron diferencias estadísticamente significativas en los porcentajes de grasa corporal $\mathrm{p}<0,05$, densidad ósea $\mathrm{p}<0,05$ y masa muscular $(\mathrm{p}<0,05)$; en la tabla 2 se muestra el comparativo de esta composición y el valor $\mathrm{p}$ correspondiente en la prueba de hipótesis.

Tabla 3

Adultos mayores, Tunja, Colombia. Valores promedio, desviación estándar y valor $\mathrm{p}$ del

Senior Fitness Test, Test de Ästrand y dinamometría en ambos grupos de adultos mayores

\begin{tabular}{lccc}
\hline \multicolumn{1}{c}{ Prueba } & $\begin{array}{c}\text { Adulto mayor } \\
\text { ciclista } \\
(\mathrm{M} \pm \mathrm{Ds})\end{array}$ & $\begin{array}{c}\text { Adulto mayor } \\
\text { sedentario } \\
(\mathrm{M} \pm \mathrm{Ds})\end{array}$ & $\mathrm{p}$ (t de student) \\
\hline $\begin{array}{l}\text { Senior Fitness Test (SFT) } \\
\text { Sentarse y levantarse } \\
\text { de una silla (\# rep) }\end{array}$ & $18 \pm 3,2$ & $17,54 \pm 4,71$ & 0,79 \\
$\begin{array}{l}\text { Levantarse, caminar } \\
\text { y volver a sentarse } \\
\text { (seg) }\end{array}$ & $4,36 \pm 05$ & $4,20 \pm 0,61$ & 0,53 \\
$\begin{array}{l}\text { Flexión de cadera } \\
\begin{array}{l}\text { Curl de brazo } \\
\text { dominante }\end{array}\end{array}$ & $-1,77 \pm 4,6$ & $-7,27 \pm 7,1$ & 0,06 \\
$\begin{array}{l}\text { Juntar las manos } \\
\text { espalda }\end{array}$ & $24 \pm 3,4$ & $22 \pm 4,12$ & 0,22 \\
\hline
\end{tabular}

Test de Ästrand (prueba submaximal)

\begin{tabular}{lccc}
\hline Prueba de esfuerzo (en & $188,63 \pm 35,99$ & $154,54 \pm 26,96$ & 0,021 \\
watts) & $156,0 \pm 10,03$ & $151,27 \pm 11,41$ & 0,31 \\
$\mathbf{F C}_{\text {submáxima }}(\mathbf{l} \cdot \mathbf{m i n})$ & $28,75 \pm 4,19$ & 0,04 \\
$\mathbf{V O}_{\text {submáximo }}(\mathrm{mlO} 2$. & $32,58 \pm 4,17$ & & \\
$\left.\mathrm{~kg}^{-1} \cdot \mathrm{min}^{-1}\right)$ & & $41,4 \pm 7,2$ & 0,76 \\
\hline Dinamometría & & $37 \pm 5,8$ & 0,0007 \\
\hline Mano derecha $(\mathrm{kg})$ & $41,3 \pm 6,8$ & & \\
Mano izquierda $(\mathrm{kg})$ & $26,7 \pm 6,2$ & & \\
\hline
\end{tabular}

Fuente: Elaboración propia

En la Tabla 3, se presentan los valores promedio registrados con su correspondiente desviación estándar, para cada una de las pruebas del Senior Fitness Test (SFT), así como el valor $\mathrm{p}$ obtenido en la comparación mediante la prueba t de Student, para la hipótesis nula; en la prueba de Sentarse y levantarse de una silla $\mathrm{p}=0,79$, levantarse, caminar y volver a sentarse $\mathrm{p}=0,53$, Curl de brazo dominante 0,22 y Juntar las manos espalda $\mathrm{p}=0,25$. No se encontraron 
diferencias estadísticamente significativas; en el test de Ästrand, hubo diferencias significativas en los watts consumidos en la prueba de esfuerzo $\mathrm{p}=0,021$ y el consumo promedio de Oxígeno $\mathrm{p}=0,04$, mientras que en la dinamometría fue la mano derecha $\mathrm{p}=0.76$ y mano izquierda $\mathrm{p}=0,0007$, la diferencia se registró para la mano izquierda.

En la evaluación cualitativa del desempeño de los dos grupos en las pruebas del SFT, realizada a partir de la estandarización por percentiles y extrapolando a partir de los promedios registrados se encontraron diferencias cualitativas en la flexión de la cadera, curl del brazo dominante y al juntar las manos en la espalda (Tabla 4)

Tabla 4

Adultos mayores, Tunja, Colombia .Comparación de la evaluación cualitativa basada en la categorización del nivel de funcionalidad según las normas de estandarización.

Senior Fitness Test (SFT)

\begin{tabular}{lcccc}
\hline \multirow{2}{*}{ Prueba } & \multicolumn{2}{c}{$\begin{array}{c}\text { Adulto mayor } \\
\text { ciclista }\end{array}$} & \multicolumn{2}{c}{$\begin{array}{c}\text { Adulto mayor } \\
\text { Sedentario }\end{array}$} \\
\cline { 2 - 5 } & Media & Calificación & Media & Calificación \\
\hline Sentarse Y Levantarse & 18 & Normal & 17,54 & Normal \\
$\begin{array}{l}\text { De Una Silla, } \\
\text { Levantarse Caminar y }\end{array}$ & 4,36 & Bueno & 4,2 & Bueno \\
$\begin{array}{l}\text { Volver a Sentarse } \\
\text { Flexión de cadera }\end{array}$ & $-1,77$ & Normal & $-7,27$ & Bajo \\
$\begin{array}{l}\text { Flexibilidad Brazo } \\
\text { Dominante }\end{array}$ & 24 & Bueno & 22 & Normal \\
$\begin{array}{l}\text { Juntar Las Manos } \\
\text { Espalda }\end{array}$ & $-4,36$ & Normal & $-9,18$ & Bajo \\
\hline
\end{tabular}

\section{Discusión}

La comparación de grupos registra como un significativo hallazgo, en lo que tiene que ver con la Composición Corporal, que los adultos mayores atletas registran menores porcentajes de grasa, que en promedio permite clasificar al grupo en la categoría de normalidad, mientras que el grupo de sedentarios, en promedio se ubica en la categoría de sobrepeso, acorde con las clasificaciones propuestas en diferentes estudios, en los que se reconoce que la actividad física genera beneficios significativos, evidenciables en la modificación positiva de los principales indicadores de salud de los hombres y mujeres adultos mayores, dado que el aumento en la edad se asocia casi siempre con modificaciones traducidas como obesidad, ateroesclerosisy riesgo metabólico-cardiovascular (Martins 2010; OMS, 2010; Barja de Quiroga 2014; Martin 2002). 
De la misma manera, en los resultados de la presente evaluación, se encuentra que los porcentajes de masa muscular y masa ósea son mayores en los sujetos que desarrollan actividad física de manera rutinaria, cuando se comparan con las personas que tienen un estilo de vida sedentario, dado que se reconoce que el metabolismo de recambio óseo se favorece cuando se desarrollan rutinariamente actividades físicas de tipo moderado a intenso; de la misma manera se aceptan ganancias en la masa ósea, atribuibles a la práctica continua, sistemática y programada de modalidades deportivas como el ciclo montañismo o los ejercicios aeróbicos (Vásquez 2013).

Para Rogers, et al, (1990) el consumo máximo de oxígeno en L/min, en adultos disminuye a una tasa aproximada del 10\% después de cumplidos los 25 años por cada década de vida cuando las personas adoptan un estilo de vida sedentario; con base en una extensiva revisión de la literatura, entre los 50 y los 75 años la disminución es de $15 \%$ por cada década. Se ha reportado que en atletas masters la disminución en el consumo de máximo de oxígeno es de alrededor de 5\% por década (Ästrand et al,. 1997). En el presente estudio se demostró la existencia de diferencias estadísticamente significativas en los valores de los watts o carga movilizada y en el consumo de oxígeno submaximal, a favor del grupo de practicantes de actividad física, porque en el análisis sobre absorción, transporte y consumo del volumen de oxígeno en los ciclo-montañistas registraron mejor rendimiento, es decir tienen mejor capacidad aeróbica, resultados que corroboran los supuestos anteriores. Este hallazgo reviste máxima importancia, por cuanto se sabe que a medida que avanza la edad puede ocurrir una disminución significativa de la frecuencia cardiaca, debido a cambios en la respuesta a los quimiorreceptores y baro-receptores, aumento del tono vagal, alteraciones de la célula miocárdica y sistema excito conductor, por causas correlacionadas con la edad: apoptosis, depósitos de colágeno y grasa tisular; estos fenómenos se evidencian objetivamente como disminución en el número de las células marcapasos tan notoria, que algunos autores han reportado a los 75 años una pérdida del 90\% de dichas células respecto a las presentes a los 20 años de edad (Ramos, et al, 2010).

La medición de la fuerza muscular y su correlación con el estado funcional y la capacidad de coordinación neuro-motriz ha sido ampliamente estudiada y se han encontrado altas correlaciones en las escalas de función e independencia cuando se compara la fuerza de prensión y el estado funcional de las extremidades mediante dinamometría (Hyatt, 1990); por otra parte, aunque se ha encontrado que la relación de la fuerza isométrica comparada en las dos extremidades es poco relevante, es cierto que se han encontrado diferencias, para el caso de las manos de hasta 10\%, en favor del lado dominante, y consideradas como normales (Miranda, 2007). No obstante, en este estudio se ha encontrado para las personas mayores, que existe una diferencia significativa, expresada en déficit de la mano contralateral a la dominante en quienes practican actividad física, lo cual podría traducirse como una mayor utilización de las manos derecha y/o izquierda asociadas a ejercicio físico practicado con mayor frecuencia e intensidad.

Desde una perspectiva más amplia, es importante considerar que los problemas de salud y capacidad funcional relacionados con el envejecimiento han sido objeto de profundo análisis en el medio académico dada la imperativa necesidad de explorar intervenciones individuales y 
colectivas que garantizando un mayor bienestar para estos sujetos mayores, representen costos accesibles para los prestadores de servicios de salud, sobre todo si se tiene en cuenta que desde hace ya bastante tiempo se tiene claro que el sedentarismo y la edad avanzada potencializan el riesgo de enfermedades respiratorias, cardiovasculares, metabólicas y degenerativas crónicas; evidencias científicas recientes muestra que la práctica adecuada del ejercicio físico, mejora la condición física, siendo actualmente la mejor manera de retrasar o prevenir el envejecimiento y sus consecuencias. Así el ejercicio físico trae beneficios, independientemente de la edad, el sexo, la salud o el estado físico de quien lo realiza. (Castillo, et al, 2006).

La actividad física practicada rutinariamente, mediante protocolos científicamente diseñados y específicos para edades y condiciones de salud preestablecidas, ofrecen un apreciable número de beneficios entre los que cabe destacar la disminución en la incidencia de todas las enfermedades cardiovasculares, ayuda a optimizar el balance nutricional y metabólico, retarda los fenómenos de resistencia a la insulina correlacionada con el envejecimiento, potencia la actividad hormonal osteoblástica reduciendo así la pérdida de mineral a nivel óseo y el riesgo de fracturas, favorece también el fortalecimiento muscular y mejora la coordinación motora, refuerza el sistema inmunológico, reduce significativamente el riesgo de algunos tipos de cáncer como los de mama, Colon y páncreas, reduce la fatiga y el estrés, mejora la capacidad eréctil en los varones, reduce el dolor y disminuye el consumo de analgésicos, incrementa y conserva la función cognitiva a la vez que disminuye el riesgo de Alzheimer, disminuye la prevalencia de depresión, ansiedad y trastornos del comportamiento a la vez que mejora la autoestima y la percepción de autoeficacia que favorecen la cohesión e integración social (Aparicio, et al, 2010). Por otra parte, la actividad física sostenida en edades avanzadas, se ha visto asociada con un mejor estado de salud general, ofreciendo de esta manera significativos beneficios reportados en las personas comprometidas con la práctica rutinaria de actividades de ejercitación, aún entre quienes iniciaron tardíamente esta práctica. En general, la actividad física representa efectos significativos sobre la fuerza, la flexibilidad, la capacidad aeróbica, la capacidad de caminar, el equilibrio y la disminución del deterioro mental y cognitivo, hecho este último que ha sido demostrado en ensayos controlados con muestras representativas en los que se ha demostrado efectos favorables de la práctica de ejercicio sobre la función cognitiva y física; también la actividad física se ha asociado con un aumento de la supervivencia en las personas mayores (Hamer, et al, 2014).

Pese a la contundencia de estos reportes, las evaluaciones que se han ocupado en medir la prevalencia de práctica de actividad física en adultos mayores coinciden en su mayoría en indicadores muy bajos; el diseño de las intervenciones reconocidas muestra una marcada tendencia a priorizar la práctica de ejercicios aeróbicos, restando significado a la ejercitación tendiente a mejorar la fuerza y la coordinación motriz (Weisser, 2009). Si bien en el presente estudio no se puede ser concluyente sobre las diferencias en la competencia de adaptación psicomotora, si se ha podido evidenciar en la literatura que el riesgo de sufrir caídas o traumatismos como consecuencia de un déficit en la coordinación neuro y senso motriz es menor en los adultos mayores que desarrollan actividad física de manera rutinaria (Toraman \& Yildirim 2010) de estas premisas se desprende el hecho de un marcado 
interés de la comunidad médica por establecer patrones de referencia con esta población, a la vez que explora los alcances de los cambios positivos producto de la práctica de actividad física, pues, pues al lograr mejor control del equilibrio, más fuerza de las piernas, mayor resistencia aeróbica y agilidad se mejora sustancialmente la autosuficiencia y la autoestima a la vez que se disminuye el riesgo de caídas y traumatismos difíciles de tratar y muy costosos. Ochoa, et al, (2015) realizaron un estudio sobre ejercicio acuático en la autonomía funcional de un grupo de adultas mayores, con una media de edad de 67.5 \pm 5.4 años, programa de intervención de 12 semanas de ejercicio acuático cinco veces por semana, 30 minutos, con una frecuencia cardiaca de reserva de 40 a 50\% (1-6 semana) y un aumento de la carga de 50 a 60\% (7-12 semana); para evaluar la autonomía funcional los investigadores utilizaron el protocolo del Grupo de Desarrollo Latinoamericano de Madurez (GDLAM); los resultados mostraron que hubo mejoría significativa $(\mathrm{p}<0,05)$ al comparar la interacción intergrupo y las mediciones en el test de caminar 10 metros $(10 \mathrm{~mW})(\mathrm{p}=0,001)$ y el índice de GDLAM (GI) $(\mathrm{p}=0,012)$, además los cambios porcentuales mostraron mejoras en los cinco componentes de (GDLAM) y (GI)\#. Estos resultados no son comprables con los de la presente investigación porque las muestras son diferentes: la de Ochoa, et al,. (2015) es en mujeres mientras este trabajo es en hombres ciclo montañistas y sedentarios, así mismo las baterías de pruebas igualmente, la primera es el índice de GDLAM (GI) y la de este trabajo es la batería de pruebas del Senior Fitness Test (SFT).

Existe en los sistemas de salud una gran preocupación por el impacto que a futuro representa el previsible aumento de la franja de población mayor de 65 años (Vidarte, 2012); es así como desde la perspectiva conceptual de la Atención Primaria en Salud, ya se han iniciado esfuerzos significativos para impulsar y evaluar el impacto de intervenciones en procura de la adopción de la práctica rutinaria de actividad física específicamente diseñada para cada grupo etario dentro del rango de edad de las personas adultas mayores; exploraciones preliminares muestran que con adecuada motivación y programación, aumenta significativamente la frecuencia de la actividad física, la fuerza del tren inferior y de la resistencia aeróbica. Recomiendan su aplicación y la adopción e implementación de programas que busquen aumentar la actividad física y el fitness en el ámbito de la atención primaria, para los adultos mayores (Purath, 2013).

\section{Conclusiones}

Se encuentra que los adultos mayores que dedican más tiempo a la práctica de actividades físicas como el ciclo montañismo, tienen una mejor capacidad aeróbica, desarrollan mayor potencia muscular y disminuyen la posibilidad de padecer diversas patologías producto del sedentarismo, alcanzan mejor aptitud funcional y coordinación motora, por ende, podrían disminuir los riesgos de salud en el plano cardiovascular, neurológico y del trauma, razón por la cual se reafirma la importancia de promover la reducción del sedentarismo, a la vez que la de estimular y facilitar la práctica de actividad física adecuada a la edad, de una manera rutinaria y persistente, con el fin de preservar la aptitud funcional y el rendimiento en la ejecución de las tareas cotidianas.

Revista MHSalud® (ISSN: 1659-097X) Vol. 13. No. 2. Febrero-Agosto, 2017 


\section{Agradecimientos}

A los adultos mayores: sedentarios y practicantes de ciclo montañismo por su participación voluntaria en el presente estudio.

Conflicto de intereses. Ninguno Declarado.

\section{Referencias Bibliográficas}

Aparicio, V., Carbonell, A., \& Delgado M. (2010). Beneficios de la actividad física en personas mayores. Revista Internacional de Medicina y Ciencias de la Actividad Física y el Deporte. 10(40),556-576. Recuperado de http://cdeporte.rediris.es/revista/revista40/ artbeneficios $181 . \mathrm{htm}$

Astrand P., \& Rodahl K. (1992). Fisiología del trabajo físico (3ª edición). Argentina: Médica Panamericana.

Ästrand, P.O., Bergh, U., \& Kilbom, A. (1997). 33 Años de Seguimiento del Consumo de Oxígeno Pico y variables relacionadas de antiguos Estudiantes de Educación Física. PubliCE Premium. Recuperado de http://g-se.com/es/fisiologia-del-ejercicio/articulos/33-anosde-seguimiento-del-consumo-de-oxigeno-pico-y-variables-relacionadas-de-antiguosestudiantes-de-educacion-fisica-307

Barja de Quiroga, G. (2014). Envejecimiento y obesidad. Anales de la Real Academia

Nacional de Farmacia. Recuperado de https:/www.analesranf.com/index.php/mono/ article/viewFile/1500/1563

Bärtsch, P. and Saltin, B. (2008). General introduction to altitude adaptation and mountain sickness. Scandinavian Journal of Medicine \& Science in Sports, 18: 1-10. doi:10.1111/ j.1600-0838.2008.00827.x

Campo, R. (2006). Factores asociados a la condición funcional del adulto mayor (Tesis de Posgrado ). Universidad de Costa Rica, Costa Rica. Recuperado de http://ccp.ucr.ac.cr/ creles/pdf/RonaldOcampo-tesis.pdf

Carlson, S., Fulton, J., Pratt, M., Yang, Z. \& Adams, EK. (January-Febrary,2015). Inadequate Physical Activity and Health Care Expenditures in the United States. Progress in Cardiovascular Diseases. 5(7), 315-323. doi: http://dx.doi.org/10.1016/j. pcad.2014.08.002

Castillo-Garzón, M. J., Ruiz, J. R., Ortega, F. B., \& Gutiérrez, Á. (2006). Anti-aging therapy through fitness enhancement. Clinical Interventions in Aging, 1(3), 213-220. doi: https:// doi.org/10.2147/ciia.2006.1.3.213 
Center for Disease Control and prevention [CDC] (2008). Physical activity Guidelines for Americans 2008:Saving Lives, Protecting People. Washington: U.S. Department of Health and Human Services. Recuperado de https://health.gov/paguidelines/pdf/paguide.pdf

Chávez, J., Lozano, M., Lara, A., \& Velásquez, O. (2004). La actividad física y el deporte en el adulto mayor, bases fisiológicas. Instituto Mexicano de Seguro Social. México DF: Masson Doyma México. Recuperado de http:/www.salud.gob.mx/unidades/cdi/ documentos/DOCSAL7516.pdf

Cooper, R., Kuh, D., \& Hardy, R., (2010) Objectively measured physical capability levels and mortality: systematic review and meta-analysis. BMJ,341: c4467. p 12. Recuperado de http://www.bmj.com/content/341/bmj.c4467.pdf\%2Bhtml

De Rosnay, J., (1988) Qué es la vida. Biblioteca Científica Salvat. Barcelona.

Del Pozo, J., Magaña, M., Ballesteros, M., Porras, M., Rodríguez, E., \& Navas, P., (junio,2013) Influencia de la capacidad funcional sobre el perfil lipídico, daño muscular y perfil bioquímico en personas mayores no institucionalizadas. Revista Andaluza de Medicina del Deporte. 6(2): 57-65. doi: https://doi.org/10.1016/S1888-7546(13)70036-4

Gomes, R., Boscher, J., Oliveira, K., Bauerfeldt, R., Da Silva, J., \& Martin Dantas, E. (2002). Efectos del entrenamiento de fuerza en la flexibilidad de mujeres mayores. Science and Sports. 17(3), 109-116. doi: https://doi.org/10.1016/S0765-1597(02)00135-1

Grøntved, A., \& Hu, F.B., (june, 2011). Television viewing and risk of type 2 diabetes, cardiovascular disease, and all-cause mortality: a meta-analysis. Journal of American Medicine Association, 305 (23), 2448-2455. doi: https://doi.org/10.1001/jama.2011.812

Hamer, M., Lavoie, K. \& Bacon, S,L., (2014). Taking up physical activity in later life and healthy ageing: the English longitudinal study of ageing. British Journal of Sports Medicine, 48 (3), 239-243. doi: https://doi.org/10.1136/bjsports-2013-092993

Hamer, M., \& Stamatakis, E. (2013) Screen-Based Sedentary Behavior, Physical Activity, and Muscle Strength in the English Longitudinal Study of Ageing. PLoS ONE, 8(6): e66222, pág 5.

Hyatt, R., Whitelaw, M., Bhat, A., Scott, S., \& Maxwell, J.D. (1990) Association of Muscle Strength with Functional Status of Elderly People. Oxford Journals Medicine. 19 (5), 330336. doi: https://doi.org/10.1093/ageing/19.5.330

Hoehn, A., Mullenbach, M., \& Fountaine, C. (2015). Actual Versus Predicted Cardiovascular Demands in Submaximal.Cycle Ergometer Testing. International Journal of Exercise Science 8(1):4-10. 
Mahoney FI \& Barthel D (1965). Functional evaluation: The Barthel Index. Maryland State Medical Journal. 14,56-61. Recuperado de: http://www.strokecenter.org/wp-content/ uploads/2011/08/barthel_reprint.pdf

Martin, E., \& Gomes de Souza, R. (2005). GDLAM'S protocol of functional autonomy evaluation. Fitness and Performance Journal, 3 (3), 175-181. Recuperado de http://www.fpjournal.org. br/painel/arquivos/1455-7_Protocolo_GDLAM_Rev3 2004_Ingles.pdf

Martín, V., Gómez, JB., \& Antoranz, MJ., (2001) Medición de la grasa corporal mediante impedancia bioeléctrica, pliegues cutáneos y ecuaciones a partir de medidas antropométricas. Análisis comparativo. Revista Española de Salud Pública, 75 (3): 221-236. doi: https://doi. org/10.1590/s1135-57272001000300006

Martín, V., Gómez, J.B., Gómez, A., \& Antoranz, M,J. (2002) Grasa corporal e índice adiposo - muscular estimados mediante impedanciometría en la evaluación nutricional de mujeres de 35 a 55 años. Revista Española de Salud Pública. 76 (6), 723-734. doi: https://doi. org/10.1590/S1135-57272002000600008

Martins, R., Verissimo, M.T., Coelho, E., Silva, M.J., Cumming, S.P., \& Teixeira, A.M. (2010) Effects of aerobic and strength-based training on metabolic health indicators in older adults. Lipid in health and Disease, 9:76. doi: https://doi.org/10.1186/1476-511X-9-76

Milanović, Z., Pantelić, S., Trajković,N., Sporiš, G., Kostić, R., \& James, N., (2013) Age-related decrease in physical activity and functional fitness among elderly men and women. Clinical Interventions in Aging. 8,549-556. doi: https://doi.org/10.2147/CIA.S44112

Miranda M. (2007) Análisis dinamométrico de la mano: valores normativos para la población española (Tesis de doctoral). Facultad de Medicina. Universidad Complutense. Madrid, España. Consulta: 16-01-2014. Recuperado de http://eprints.ucm.es/12341/

Moreno, A., (2005) Incidencia de la Actividad Física en el adulto mayor. Revista Internacional de Medicina y Ciencias de la Actividad Física y el Deporte. 5(19), 222-237. Recuperado de http://cdeporte.rediris.es/revista/revista20/artvejez16.htm

Ochoa, P., Hall, J., Paredones, A., \& Martin Dantas, E. (2015). Effect of periodized water exercise training program on functional autonomy in elderly women. Nutrición Hospitalaria, 31(1);351-356. Recuperado de http://www.aulamedica.es/nh/pdf/7857.pdf

Organización Mundial de la Salud [OMS]. (2010). Recomendaciones mundiales sobre actividad física para la salud. Ginebra: Biblioteca OMS. Recuperado de http://www.who.int/ dietphysicalactivity/publications/9789241599979/es/ 
Porter, M. (2006). Power training for older adults. Applied Physiology, Nutrition, and Metabolism, 31(2), 87-94. doi: https://doi.org/10.1139/h05-034

Purath, J., Keller, C.S., MacPherson, S., \& Ainsworth, B. (may-june,2013). A randomized controlled trial of an office-based physical activity and physical fitness intervention for older adults. Geriatric Nursing. 34 (3); 204-211. doi: http://dx.doi.org/10.1016/j. gerinurse.2013.02.012

Ramos, C., Santos, A., Ochoa, M., Peña, N., Ramos, R., \& Ramos-Pupo, R. (2010) Comportamiento fisiológico cardiorespiratorio en el adulto mayor durante el ejercicio físico. Correo medico científico de Holguin, 14(3),1-12. Recuperado de http:/www.cocmed.sld.cu/no143/pdf/ no143rev01.pdf

Rikli, R., \& Jones, C. (1999a) The development and validation of a functional fitness test for community residing older adults. Journal of Aging and Physical Activity. 7, 129-161. doi: $\underline{\text { http://dx.doi.org/10.1123/japa.7.2.129 }}$

Rikli, R. \& Jones, C. (1999b) Functional fitness normative scores for community-residing adults, ages 60-94. Journal of Aging and Physical Activity. 7, 160-179. doi: https://doi.org/10.1123/ japa.7.2.162

Rogers, M.A., Hagberg, J.M., Martin, W.H., Ehsani, A.A., \& Holloszy, J.O. (1990). Decline in VO2 max with aging in master athletes and sedentary men. J. Appl. Physiol. 68(5), $2195-$ 2199. Recuperado de https://www.ncbi.nlm.nih.gov/pubmed/2361923

Salazar, J., Ramírez, J., \& Chaparro, D. (2014). Revisión sistemática sobre el impacto de la actividad física en los trastornos de la marcha en el adulto mayor. Apunts. Educación Física y Deportes.118(4),30-39. doi:.https://doi.org/10.5672/apunts.2014-0983.es.(2014/4).118.03

Toraman, A., \& Yildirim, U.N., (2010). The falling risk and physical fitness in older people. Arch Gerontol Geriatr. 51(2),222-226.

Universidad Pedagógica y Tecnológica de Colombia. Tunja, Boyacá. [UPTC]. Hacia una Universidad Saludable: Caracterización de la comunidad universitaria UPTC. Recuperado de http://ups.uptc.edu.co/SistemaUPS/Documentos/CaracterizacionUPTC2012.pdf

United Nations Organization [UNO] (2005). World Population Prospects: The 2004 Revision. New York: United Nation.

United States of America. The Secretary of health and human services. 2008 Physical activity guidelines for Americans. Government of the United States. Washington DC. ODPHP publication No. U0036. October, 2008 Recuperado de https://health.gov/paguidelines/pdf/ paguide.pdf 
Vásquez, A., Wanden, C., \& Sanz, J. (2013). Ejercicio físico y suplementos nutricionales; efectos de su uso combinado en las personas mayores de 65 años; una revisión sistemática. Nutrición hospitalaria. 28 (4),1077-1084.

Vidarte, J.A., Quintero, M.V., \& Herazo, Y. (julio-diciembre,2012) Efectos del ejercicio físico en la condición física funcional y la estabilidad en adultos mayores. Hacia la promoción de la salud. 17 (2), 79-90. Recuperado de http://www.redalyc.org/articulo.oa?id=309126826006

Weisser, B., Preuss, M., \& Predel, H.G. (2009) Physical activity for prevention and therapy of internal diseases in the elderly. MedKlin, 104(4):296-302. doi: https://doi.org/10.1007/ $\underline{\text { s00063-009-1055-1 }}$

Recepción: 19 de setiembre del 2016

Corrección: 14 de diciembre del 2016

Aceptación: 16 de enero del 2017

Publicación: 31 de enero del 2017

1 Licenciado, Maestrante en Pedagogía de la Cultura Física. Universidad Pedagógica y Tecnológica de Colombia. Tunja, Colombia. Docente, Fundación Universitaria Juan de Castellanos. e-mail: gioquinmon@hotmail.com

2 Licenciado. Msc. en Pedagogía de la Cultura Física. Universidad Pedagógica y Tecnológica de Colombia. Tunja, Colombia. Docente -Investigador Fundación Universitaria Juan De Castellanos. e-mail: vic.melgarejo@live.com

3 Médico. MSc en Epidemiología. Docente Universidad Pedagógica y Tecnológica de Colombia. Tunja, Colombia. e-mail: $\underline{\text { Juan.Ospina@Uptc.Edu.Co }}$ 